\title{
Esophageal transit scintigraphy in systemic sclerosis
}

\author{
Marek Chojnowski ${ }^{1}$, Małgorzata Kobylecka², Marzena Olesińska ${ }^{3}$ \\ ${ }^{1}$ Department of Nuclear Medicine, Central Clinical Hospital, Warsaw, Poland \\ 2Department of Nuclear Medicine, Warsaw Medical University, Warsaw, Poland \\ ${ }^{3}$ Department of Connective Tissue Diseases, National Institute of Geriatrics, Rheumatology and Rehabilitation, Warsaw, Poland
}

\begin{abstract}
Systemic sclerosis is a rare connective tissue disease, distinctive features of which are fibrosis and microangiopathy. The esophagus is one of the most commonly involved internal organs. Most patients experience dysphagia, difficulties in swallowing and gastro-esophageal reflux. However, in up to one third of cases, the initial onset of esophageal disease may be clinically silent. There are several diagnostic modalities available for assessing both morphological and functional abnormalities of the esophagus. If structural abnormalities are suspected, endoscopy is the method of choice. Functional evaluation is best achieved with manometry. Both endoscopy and manometry are invasive techniques, with low patient acceptance. Barium-contrast study is well tolerated, but qualitative assessment of functional abnormalities is imprecise. Esophageal scintigraphy is an easy, non-invasive, sensitive and specific diagnostic modality. It can detect esophageal dysfunction even in asymptomatic patients. In patients already diagnosed with systemic sclerosis, scintigraphy is useful in evaluating severity and progression of the disease.
\end{abstract}

Key words: scintigraphy, systemic sclerosis, esophagus, gastrointestinal tract.

\section{Introduction}

Systemic sclerosis (SSc) is a rare connective tissue disease of unknown etiology. Its hallmarks are progressive fibrosis and microangiopathy leading to skin thickening, as well as dysfunction of multiple internal organs [1]. The involvement of the gastrointestinal (GI) tract, primarily the esophagus, is observed in about $90 \%$ of patients [2]. Difficulties with swallowing can be the first manifestation of SSc, although in some patients an ongoing esophageal disease may be asymptomatic for a long time. Since esophageal dysmotility is prevalent in SSc patients, its reliable detection is essential not only for initial diagnosis, but also for monitoring progression of the disease. Among different modalities dedicated to upper Gl tract diagnostics, esophageal transit scintigraphy is of particular interest, being a safe, easy and well-tolerated technique.

\section{Gastrointestinal involvement in systemic sclerosis}

The principal pathogenetic mechanisms of SSc are increased synthesis of extracellular matrix followed by progressive fibrosis and endothelial damage of small vessels caused by as-yet-unknown factors. Both these phenomena are well documented in the case of hands, where fibrosis is seen as skin thickening with finger contractures, and microangiopathy manifests as non-healing digital ulcerations with necrosis [3].

Similar processes are observed in the course of internal organ involvement, including the Gl tract. Microangiopathy causes tissue hypoperfusion and ischemia, which lead to atrophy of the neural fibers and autonomic dysfunction. Muscarinic-3 acetylcholine receptor autoantibodies, detected in the majority of patients with Gl involvement, may directly disrupt cholinergic neurotransmission in the smooth muscles [4]. As a consequence, esophageal reflex- 
es are weakened or suppressed, greatly impairing peristalsis. Simultaneously, smooth muscle cells are replaced by proliferating fibroblasts and deposits of collagen fibers which thicken and harden GI tract walls [5]. Telangiectases may form in the mucosal membrane, especially of the stomach, causing bleeding and ulcerations.

The abovementioned pathologies are most prominent in the lower esophagus, slowing the passage of digestive contents from the esophagus to the stomach, or even leading to gastrointestinal stasis.

A concurrent decrease in lower esophageal sphincter pressure leads to the occurrence of gastroesophageal reflux with induction of intestinal metaplasia (Barrett's esophagus) and increased risk of developing adenocarcinoma [4]. Involvement of the stomach, small intestine and colon is less prevalent, affecting $20-70 \%$ of patients with SSc [5].

Dysmotility and structural damage of the Gl tract typically manifest as dysphagia, regurgitation, bloating, heartburn, and in severe cases aspiration of digestive contents, intestinal obstruction or malabsorption syndrome [2].

\section{Diagnostic techniques for evaluating esophageal motility}

Several diagnostic modalities are useful for both morphological and functional assessment of the esophagus [6]. One of the most commonly performed procedures is upper Gl endoscopy (esophagogastroduodenoscopy). When stenosis, bleeding or malignancy is suspected, endoscopic investigation is considered the gold standard. However, this method has low sensitivity in detecting functional disorders, and its usefulness in early diagnosis of SSc is limited.

Manometry is a procedure dedicated to functional evaluation of the esophagus. A probe equipped with multiple pressure detectors is capable of measuring the strength and amplitude of muscular contractions, both at rest and during swallowing. In SSc patients, manometry most commonly indicates hypomotility, that is low amplitude of muscle contractions, infrequent contractions and overall ineffectiveness of peristalsis.

Both endoscopy and manometry are invasive techniques, with low patient acceptance. Especially manometry is poorly tolerated, since it requires insertion of the probe through the nasal cavity.

Fluoroscopic study with barium swallow is useful in assessing upper Gl tract morphology and, to some extent, esophageal motility [7]. During the test, barium contrast medium fills the lumen of the GI tract, outlining its walls. Peristalsis is evaluated by means of real-time fluoroscopic image acquisition. Barium swallow study is well tolerated, but precise qualitative assessment of functional abnormalities is only approximate and has low reproducibility.

\section{Esophageal transit scintigraphy}

Esophageal scintigraphy is designed to examine the motility and speed of peristalsis [8]. The patient should fast for at least 4 hours before the test. The scintigraphy consists of swallowing a small amount of radiotracer (usually technetium-99m labeled liquid) followed by immediate image acquisition by a gamma camera. The images are registered in two phases. The dynamic study involves acquiring one image of the esophagus every 0.5 second for 2-3 minutes. After another 2 minutes, a static image of the esophagus and upper stomach is obtained, to detect late stagnation of the radiotracer in the esophagus.

Computer-assisted analysis of images obtained in the dynamic phase is the basis of qualitative, objective assessment of esophageal motility and peristalsis. The upper GI tract is divided into four regions of interest (ROI), corresponding to the upper, middle and lower esophagus and upper part of the stomach. From these ROI, time-activity curves are created, which show how fast the radiotracer travels through successive parts of the upper Gl tract (Figs. 1, 2). Esophageal transit time (ETT) and esophageal emptying time (EET) are useful and repeatable parameters that can be calculated from time-activity curves. Esophageal transit time is defined as the time between the appearance of $50 \%$ of radioactivity in the upper esophagus and clearance of 50\% of activity from the entire esophagus. By analogy, EET is defined as the time between the appearance of 50\% of radioactivity in the upper esophagus and clearance of $100 \%$ of activity from the entire esophagus [9].

Table I compares ETT and EET values in patients with primary Raynaud's phenomenon (RP), SSc and healthy volunteers. Retention fraction is yet another parameter for detecting esophageal dysmotility. It is an activity retained in the esophagus at a given time after the swallow divided by the maximal activity and expressed as a percentage. Retention fraction at 90 seconds $\left(R_{90}\right)$ seems to be most useful, as reported by Nakajima et al. [10].

Esophageal transit study is routinely performed in the supine position in order to eliminate the effect of gravity on the radioactive bolus. Some authors, however, postulate that position does not alter the results of the study. In patients with severe dysphagia and high risk of aspiration, scintigraphy can be performed in an upright, sitting position (Fig. 3). 


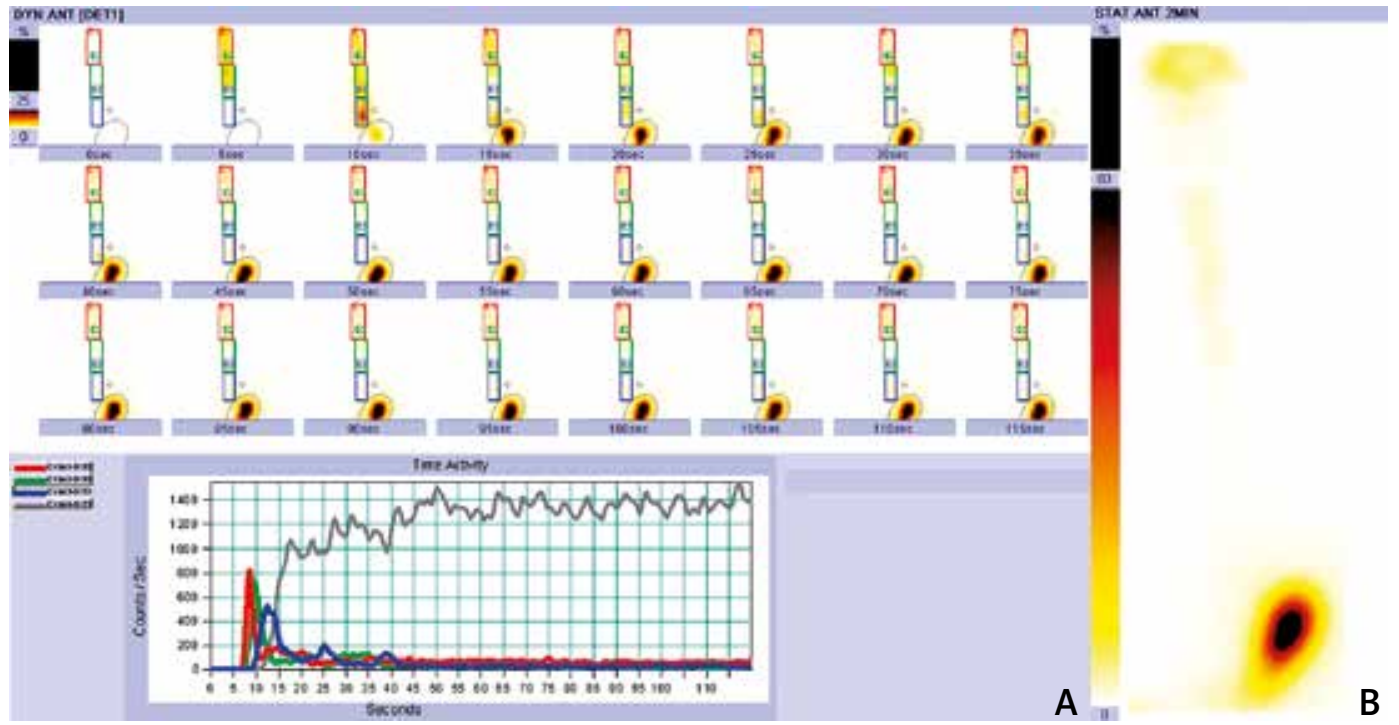

Fig. 1. Normal esophageal transit scintigraphy - radiotracer reaches the stomach within first 5 seconds, after 40 seconds all the radioactivity is cleared from the esophagus (A). Static image obtained after 2 minutes shows radiotracer in the stomach. Clinically insignificant traces of radioactivity are also visible in the oral pharynx and upper esophagus (B).

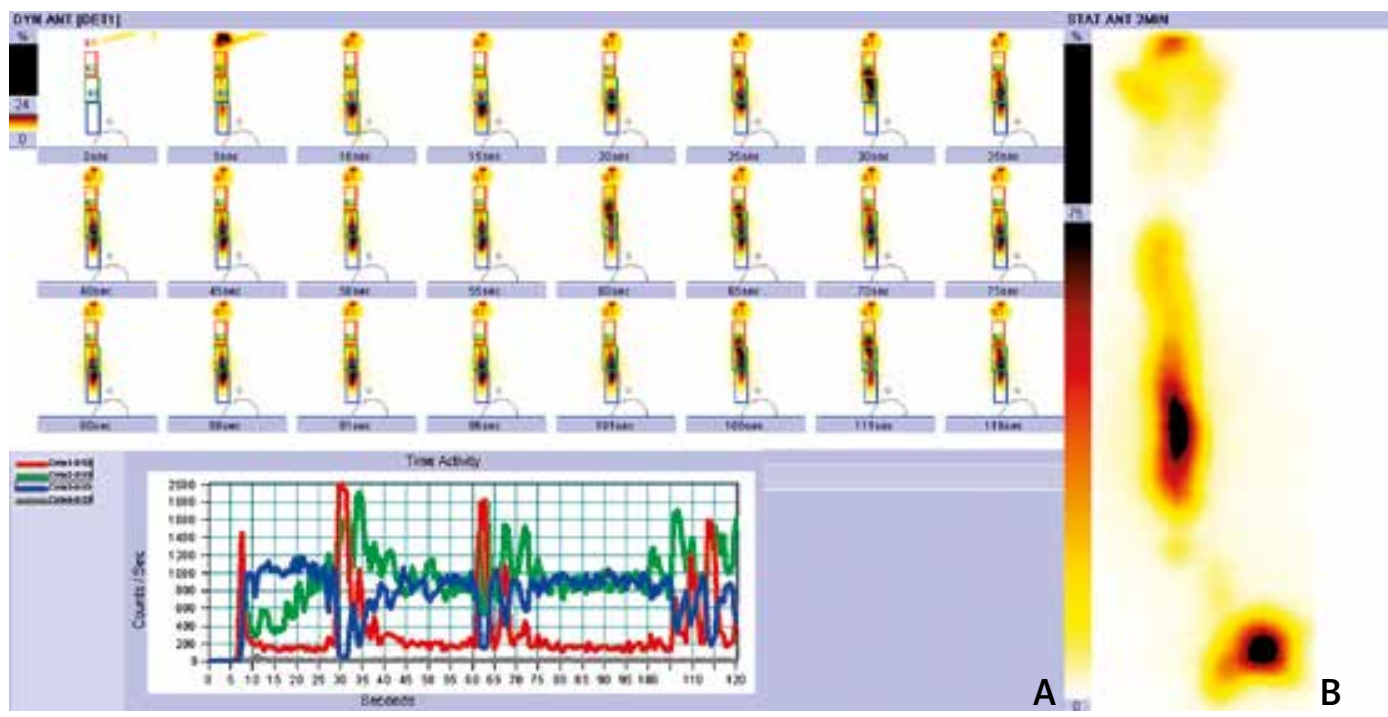

Fig. 2. Esophageal transit scintigraphy in patient with SSc - retention of the radiotracer in middle and lower esophagus, no radioactivity visible in the stomach, prominent waves of retroperistalsis (A). Static image obtained after 2 minutes shows only partial clearance of radiotracer to the stomach, with stagnation in the middle and lower esophagus (B).

Esophageal scintigraphy has many advantages, primarily precise qualitative assessment of esophageal function, short duration, safety and high patient acceptance. Moreover, the dose of radiation absorbed throughout scintigraphy is several times lower than during the barium swallow study [11].
The main disadvantage is rudimentary visualization of anatomy, which makes it impossible to reliably detect structural pathologies. For this reason, scintigraphy cannot replace endoscopy or barium swallow when morphological abnormalities, e.g. stenosis or tumor, are suspected. Despite high sensitivity and specificity of 
Table I. Comparison of esophageal transit time and esophageal emptying time in healthy individuals, patients with Raynaud's phenomenon and systemic sclerosis (after Bestetti et al. [9], with modifications)

\begin{tabular}{|cccc|}
\hline & Healthy controls & Raynaud's phenomenon & Systemic sclerosis \\
\hline ETT (s) & & & 16 \\
supine & 7 & 9 & 19 \\
upright & 7 & 9 & 362 \\
\hline EET (s) & 40 & 163 & 269 \\
supine & 43 & 106 & \\
upright & & & \\
\hline
\end{tabular}

ETT - esophageal transit time; EET - esophageal emptying time; $s$ - seconds

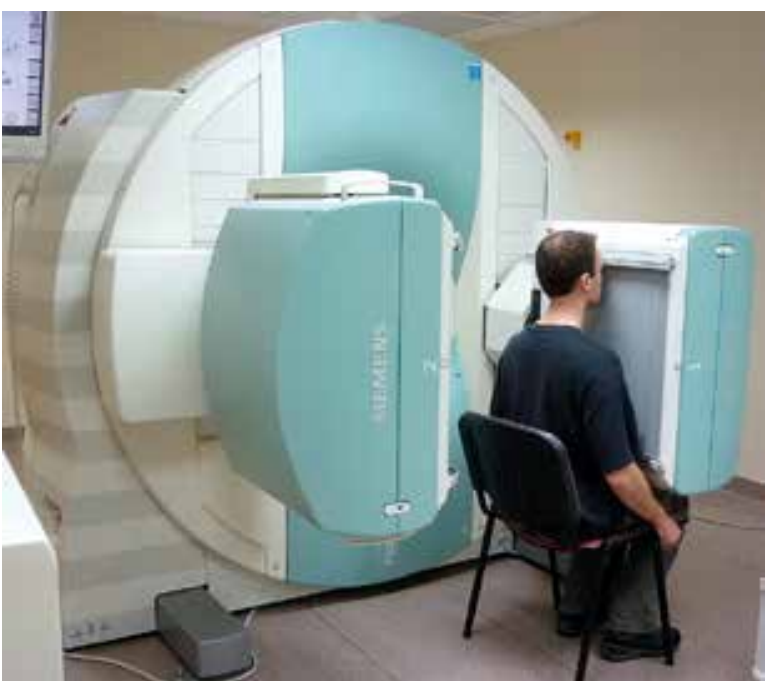

Fig. 3. Gamma camera configured for esophageal scintigraphy in upright position.

esophageal scintigraphy, no universal study protocol has been accepted so far. In different departments, the details of the scintigraphic procedure may vary significantly, e.g. a solid or semi-solid radiotracer can be used, or the scintigraphic images can be registered after several successive swallows [12]. Also quantitative parameters may differ, for example esophageal transit time is often defined as the interval between appearance of $10 \%$ of radioactivity and clearance of $90 \%$ of radioactivity from the entire esophagus [13].

\section{Significance of esophageal transit study in systemic sclerosis}

In SSc patients, the esophagus is one of the most commonly affected internal organs. In the majority of cases, symptoms of dysphagia and gastroesophageal reflux are present at early stages of the disease, although in up to $30-40 \%$ of patients esophageal involvement may be clinically silent for a long time. There is no general agreement which diagnostic test should be performed as an initial screening in asymptomatic cases. Considering the pathogenetic mechanisms of SSc, at the very beginning of esophageal disease functional abnormalities are dominant, with slight or no morphological changes. As it is, the screening method should focus on evaluating motility and peristalsis of the GI tract. In this aspect, esophageal scintigraphy seems more appropriate than the uncomfortable and poorly tolerated manometric study, especially because sensitivity and specificity of both diagnostic modalities are comparable $[8,13,14]$.

Assessment of dysphagia is useful in monitoring patients with high risk of developing SSc or related disorders. The 'early' capillaroscopic scleroderma pattern observed in RP patients can preexist for many years without any other symptoms, before the full onset of SSc [15]. A similar phenomenon may occur in esophageal dysmotility. Bestetti et al. [9] compared scintigraphic values of ETT and EET in patients with primary RP, SSC and in healthy controls. Compared to non-affected individuals, all SSc patients had both parameters greatly increased. In the RP group, ETT and EET were lower than in SSc, but significantly higher in comparison with healthy controls. Nakajima et al. [10] demonstrated a correlation of esophageal dysmotility detected in scintigraphy with disease severity in SSc. No prolonged retention was detected in patients with suspicion of SSc. In the case of definite SSc diagnosis, more severe impairment of peristalsis was reported in patients with diffuse SSc and extensive skin involvement (> 10 points in modified Rodnan skin score) than in individuals with limited SSc [10, 16]. Kinuya et al. [17] noted a negative correlation between diffusing capacity of the lung for carbon monoxide $\left(\% \mathrm{D}_{\mathrm{LCO}}\right)$ and esophageal hypomotility. This suggests the possibility of indirect assessment of interstitial lung disease severity, based on the degree of esophageal stagnation [17]. Finally, scintigraphy, being a simple and non-invasive study, seems to be an optimal diagnostic 
technique for routine monitoring of esophageal disease progression, as suggested by Vischio et al. [18].

\section{Summary}

Esophageal transit scintigraphy is a sensitive, specific, safe and well-tolerated procedure. Despite the fact that it cannot substitute endoscopy or barium swallow study in detection of morphological abnormalities, it is an easy and efficient technique for functional evaluation. In SSc, esophageal scintigraphy is an attractive screening tool during initial diagnosis and a simple and repeatable method for monitoring progression of the disease.

\section{Acknowledgements}

The presented paper is a part of the first author's PhD thesis.

The authors declare no conflict of interest.

\section{References}

1. Elhai M, Avouac J, Kahan A, Allanore Y. Systemic sclerosis: Recent insights. Joint Bone Spine 2015; 82: 148-153.

2. Pulkowski G, Budzyński J, Kłopocka M, et al. Digestive tract in patients with systemic sclerosis. Reumatologia 2006; 44: 95-101.

3. Hughes M, Ong VH, Anderson ME, et al. Consensus best practice pathway of the UK Scleroderma Study Group: digital vasculopathy in systemic sclerosis. Rheumatology (Oxford) 2015; 54: 2015-2024.

4. Wielosz E, Borys O, Zychowska I, Majdan M. Gastrointestinal involvement in patients with systemic sclerosis. Pol Arch Med Wewn 2010; 120: 132-136.

5. Clements PJ, Becvar R, Drosos AA, et al. Assessment of gastrointestinal involvement. Clin Exp Rheumatol 2003; 21 (Suppl. 29): S15-S18.

6. Kuo P, Holloway RH, Nguyen NQ. Current and future techniques in the evaluation of dysphagia. J Gastroenterol Hepatol 2012; 27: 873-881.

7. Saleh CM, Smout AJ, Bredenoord AJ. The diagnosis of gastro-esophageal reflux disease cannot be made with barium esophagograms. Neurogastroenterol Motil 2015; 27: 195-200.

8. Pitrez EH, Bredemeier M, Xavier RM, et al. Oesophageal dysmotility in systemic sclerosis: comparison of HRCT and scintigraphy. Br J Radiol 2006; 79: 719-724.

9. Bestetti A, Carola F, Conciato L, et al. Esophageal scintigraphy with a semisolid meal to evaluate esophageal dysmotility in systemic sclerosis and Raynaud's phenomenon. J Nucl Med 1999; 40: 77-84.

10. Nakajima K, Kawano M, Kinuya K, et al. The diagnostic value of oesophageal transit scintigraphy for evaluating the severity of oesophageal complications in systemic sclerosis. Nucl Med Commun 2004; 25: 375-381.
11. Mettler F, Guibertau M. Gastrointestinal Tract. In: Essentials of Nuclear Medicine Imaging. Mettler F, Guiberteau MD (eds.). 6th ed. Elsevier, Philadelphia 2012; 261-268.

12. Maurer A. Gastrointestinal Motility. Part 1: Esophageal Transit and Gastric Emptying. J Nucl Med 2015; 56: 1229-1238.

13. Parkman HP, Maurer AH, Caroline DF, et al. Optimal evaluation of patients with nonobstructive esophageal dysphagia. Digest Dis Sci 1996; 41: 1355-1368.

14. Drane WE, Karvelis K, Johnson DA, et al. Progressive systemic sclerosis: radionuclide esophageal scintigraphy and manometry. Radiology 1986; 160: 73-76.

15. Chojnowski M, Felis-Giemza A, Olesińska M. Capillaroscopy a role in modern rheumatology. Reumatologia 2016; 54: 67-72.

16. Nakajima K, Hasegawa M, Inaki A, et al. Esophageal transit study using a sliding sum image: application to patients with probable and definite systemic sclerosis. Ann Nucl Med 2011; 25: 325-331.

17. Kinuya K, Nakajima K, Kinuya S, et al. Esophageal hypomotility in systemic sclerosis: Close relationship with pulmonary involvement. Ann Nucl Med 2001; 15: 97-101.

18. Vischio J, Saeed F, Karimeddini M, et al. Progression of esophageal dysmotility in systemic sclerosis. J Rheumatol 2012; 39: 986-991. 